\title{
APORTES DE LA EDUCACIÓN MUSICAL A LAS PERSONAS CON DISCAPACIDAD VISUAL EN LA PROMOCION DE LA AUTODETERMINACION
}

\section{CONTRIBUTIONS OF MUSICAL EDUCATION TO PEOPLE WITH VISUAL DISABILITIES IN THE PROMOTION OF SELF-DETERMINATION}

Mónica Pérez Valderrama *

Correo electrónico: monicaperezvalderrama@gmail.com

Fecha recibido: 20 - 01- 2020

Fecha de aprobación: 12 - 02 - 2020

* Magíster en Educación y Desarrollo Humano, Universidad de Manizales - CINDE. Licenciada en Música, Corporación Universitaria Adventista. Integrante del equipo investigador de Música para ver: Sistematización de la experiencia. 
Resumen

El presente artículo está basado en un trabajo investigativo, de corte cualitativo, que se aproximó al programa de educación musical Música para ver, con la intencionalidad de develar las transformaciones subjetivas de los participantes entre los años 2014 2016, para reconocer los alcances del programa y potenciar sus estrategias. El estudio fue realizado con 16 adolescentes estudiantes de música con discapacidad visual y sus familias en Medellín Colombia, y reveló los cambios de los participantes en la construcción de su subjetividad, el desarrollo de sus habilidades, proyecto de vida, vínculos afectivos y la transformación hacia un concepto positivo de la discapacidad en sus familias, a través del aprendizaje de la música. El objetivo del trabajo fue registrar los aprendizajes develados en la investigación y derivados de la reflexión de la investigadora, en torno al fomento de la autodeterminación en la práctica musical de personas con discapacidad visual. Para ello, se desglosa las características y obstáculos para el desarrollo de conductas autodeterminadas, se realiza un acercamiento a los cambios en la normatividad y en los principios de la educación musical que permitieron la inclusión en el aula de música. Finalmente, se sustenta la tesis de que la práctica musical colectiva en un ambiente educativo potencializador favorece el desarrollo de la autodeterminación en personas con discapacidad visual.

Palabras clave: Educación musical; discapacidad visual; inclusión; autodeterminación; Medellín.

\section{Abstract}

The present article is based on the research work Música para ver: Sistematización de la experiencia (Music to see: Systematization of experience); a qualitative research, which approached the music education program Música para ver intended to unveil the subjective transformations of the participants in the city of Medellin between 2014-2016, to recognize the scope of the program and to strengthen its strategies. This research was made with 16 teenage music students with visual disabilities (VD) and their families in Medellin - Colombia, revealing the changes the participants had on the creation of their subjectivity, abilities development, life's project, emotional bonds and transformation towards a positive concept of the disability in their families, all through music learning. This article aims to complement the conceptual referent of this research and create a record of the learnings unveiled from the research and derived from the researcher's consideration surrounding the increase of self-determination with the musical practice of people with visual disabilities (VD). To this effect, the research briefly breaks down the characteristics and the impediments for the development of selfdetermined behaviors, there is an approximation to the changes in the regulations and the principles of Music Education that allows a more inclusive music classes. Ultimately, the research backs the thesis of collective musical practice encouraging the development of selfdetermination in people with VD.

Keywords: Musical education, visual impairment, involvement, self-determination, Medellín. 


\section{Introducción}

Durante años, la educación musical se ha presentado como una potente alternativa para el desarrollo de capacidades humanas que favorece, a través de la interacción y las actividades motrices, cognitivas y sensoriales, el despliegue de las facultades mentales y afectivas, especialmente efectivas en el abordaje en la educación de las personas con discapacidad (Castro et. al., 2013). A pesar de ello, la inclusión de las personas con discapacidad en la educación formal y no formal musical en Colombia es un hecho reciente y aislado, con muy poca investigación y rara vez sistematizado, lo que genera vacíos conceptuales y desconcierto en los docentes de música que se enfrentan al reto de incluir personas con discapacidad en el aula.

Este desconocimiento converge en la omisión del desarrollo de la autodeterminación de las personas con discapacidad en el ejercicio musical, entendiendo la autodeterminación como la capacidad de los sujetos de planear, dirigir y ejecutar tareas de interés personal haciendo uso de la autorregulación (auto-instrucciones, auto-refuerzo), las creencias sobre su capacidad de control y eficacia, y la autoconciencia (conocimiento de sus puntos fuertes y de sus limitaciones) (Peralta y González-Torres, 2006). La discapacidad suele asociarse con la incapacidad para el ejercicio de la libertad, lo que obstaculiza la participación del sujeto con discapacidad en la toma de decisiones sobre sus intereses y su propio proceso educativo musical.

Teniendo en cuenta esta problemática, que hace notoria la importancia de promover la autodeterminación en las personas con discapacidad, se genera la pregunta que orienta el tema del presente artículo: ¿Cuáles son los aportes que la educación musical puede ofrecer a las personas con discapacidad visual para promover conductas autodeterminadas?

Para responderla, el presente texto se basa en el trabajo investigativo Música para ver: Sistematización de la experiencia, realizado en el contexto de la línea Ambientes Educativos perteneciente al Grupo de Investigación Pedagogía y Educación: Saberes, Imaginarios y Subjetividades, avalado por la Universidad de Manizales y la Fundación Centro Internacional de Educación y Desarrollo Humano (CINDE). Dicha sistematización de experiencias tuvo como objetivo develar las transformaciones subjetivas de los participantes del programa en la ciudad de Medellín entre los años 2014-2016, reconocer los alcances del programa y potenciar sus estrategias.

El estudio fue realizado con 16 adolescentes estudiantes de música con discapacidad visual y sus familias, y reveló los cambios de los participantes en la construcción de su subjetividad partiendo del aprendizaje de la música (iniciación musical a partir de las flautas dulces), específicamente en el desarrollo de habilidades que fomentan autonomía, la inclusión de la música en su proyecto de vida (oportunidades), la resignificación de las emociones (esperanza y felicidad) y los vínculos afectivos (familiaridad) y la transformación de la mirada hacia un concepto positivo de la discapacidad en las familias y cuidadores (Pérez, Ruiz y Ossa, 2019). 
En el marco de dicha sistematización, este trabajo tiene como objetivo: a) complementar el referente conceptual de la investigación al abordar la evolución del concepto de autodeterminación y su relación con la discapacidad, desde el paradigma del modelo social; y b) registrar los aprendizajes develados en la investigación y derivados de la reflexión de la investigadora, en torno al fomento de la autodeterminación en la práctica musical de personas con discapacidad visual. Para ello, desglosa brevemente las características y obstáculos a los que se enfrenta esta población para el desarrollo de conductas autodeterminadas. Posteriormente, se realiza una aproximación a los cambios en los principios de la educación musical latinoamericana y la publicación de los derechos de las personas con discapacidad, los cuales permitieron su inclusión en programas musicales no formales, dentro de los cuales se encuentra Música para ver. Finalmente, se sustenta la tesis de que la práctica musical colectiva favorece el desarrollo de la autodeterminación en niños, niñas y adolescentes con discapacidad visual en la experiencia de Música para ver, como ambiente educativo potencializador de conductas autodeterminadas, con base en la teoría de la autodeterminación desarrollada por Wehmeyer y en conversación con los aportes de Peralta y González, Verdugo y Tamarit, partiendo de los aprendizajes develados en la sistematización de experiencias y derivados de la reflexión de la investigadora.

\section{Estado del arte}

Como parte del proceso investigativo de Música para ver: Sistematización de la experiencia se realizó un rastreo de estudios e investigaciones sobre experiencias prácticas de educación musical para personas con discapacidad visual en Colombia. En el momento que se realizó la investigación (2014-2015), los estudios encontrados se enfocaban en el aprendizaje de las matemáticas (Torres y Gaviria, 2013; Correa, y Pulido, 2013; Andrade, 2013), la lecto-escritura braille (Andrade, 2013; Loaiza y Torres, 2013) y la educación física Burgos, 2013; Chacón, Camargo y Cotachira, 2014), tanto en la creación de estrategias pedagógicas como en la adaptación de currículo y el desarrollo tecnológico para abordar dichas áreas del conocimiento. Dichas investigaciones coinciden en que "el acervo investigativo en Colombia acerca de la educación de los niños, niñas y adolescentes en situación de discapacidad visual se implementa casi siempre en el ámbito escolar, orientada a la adecuación de las didácticas hacia la inclusión" (Pérez, Ruiz y Ossa, 2019, p. 11). Los resultados expusieron la ausencia de estudios en educación musical no formal enfocada a la discapacidad visual en el país.

En el momento de la construcción de este artículo (2019), las mismas categorías de búsqueda arrojaron algunos estudios, lo que puede indicar que el tema ha ido tomando un espacio en las publicaciones y ejercicios investigativos del país. En primer lugar, Castillo Castellanos, y Delgado Villamizar (2012) realizan en la Universidad Industrial de Santander una Transcripción al sistema braille de material básico para el estudio de 
la música, con el objetivo de facilitar material de estudio de la música a estudiantes ciegos de la Licenciatura en Música, reconociendo la importancia y la necesidad del uso de la musicografía braille (método de lecto-escritura musical para ciegos) en el proceso de enseñanza aprendizaje de un estudiante de música ciego. La transcripción del material está a disposición en la biblioteca de la institución.

Luego, se encontraron estudios como el de Arias Cancino (2015): Estrategias pedagógicas desde la inclusión educativa para el fortalecimiento de la expresión y la conciencia corporal para estudiantes de música con discapacidad visual, por medio del cual se diseñó e implementó la propuesta "Mirar en ausencia de luz", con el objetivo de ofrecer, a los docentes y estudiantes con discapacidad visual del programa de Licenciatura en Música de la Universidad Pedagógica Nacional, orientación en el desarrollo de la expresión corporal y el manejo del cuerpo en la escena, por medio de la sensopercepción y la eutonía. Aunque el estudio se aleja un poco del interés de esta investigación, se considera importante resaltarlo por su relación con el desarrollo artístico y el impacto visual que tiene en la audiencia el performance de una persona con discapacidad visual.

Un año después se realizaron dos publicaciones en la ciudad de Medellín. La primera de ellas, por Cano Hoyos (2016). en la Universidad Pontificia Bolivariana: Inclusión a personas con discapacidad visual en los pregrados y licenciaturas. En ella, el autor realiza una investigación en la que se cuestiona el acceso de las personas con discapacidad visual en educación musical a nivel superior en la ciudad. Entre sus conclusiones se hace reiterativo que las instituciones y docentes universitarios de las facultades de música desean admitir a estudiantes con discapacidad visual en sus universidades, pero consideran que no tienen la capacitación técnica, pedagógica ni tecnológica para la enseñanza inclusiva en el aula universitaria. Además, se insiste en la importancia de las TIC en la contribución a la inclusión de las personas con discapacidad en este programa profesional.

La otra referencia es sobre el artículo reflexivo de Suárez Román (2016), estudiante de Maestría en Música - Canto Jazz de la Universidad EAFIT, bajo el nombre de Percepción de la música en la discapacidad visual. En el escrito, el autor comparte las estrategias mentales que denomina "piano mental", "coro mental" y "orquesta mental", habilidades creadas por él para comprender los conceptos de la verticalidad de la música (en musicografía braille, toda la música se representa de manera horizontal). Estas estrategias de percepción y organización mental permitieron al autor profundizar en contrapunto, armonía y composición, y participar en ensambles sinfónico-corales. Además, comparte las situaciones cotidianas a las que se enfrentó en su calidad de estudiante de música universitario, y cómo solucionó cada obstáculo hasta lograr su título profesional.

Es importante resaltar que cuatro de los autores de estas publicaciones investigativas y académicas son personas con discapacidad visual, estudiantes de programas de música a nivel nacional. Este dato puede mostrar que conocer, comprender, escribir, 
reflexionar y publicar acerca del tema en Colombia puede ser, por ahora, interés de quienes enfrentan el reto de ser estudiantes de música con discapacidad visual. Hay que mencionar que la iniciativa de registrar sus necesidades, vacíos, herramientas y propuestas es útil para que las instituciones y docentes universitarios de los pregrados de música se pregunten también por estas necesidades, y se realicen los ajustes necesarios para lograr la inclusión en sus aulas.

Los estudios hasta aquí resumidos muestran que las últimas investigaciones en torno al tema en Colombia se dan principalmente en el nivel de pregrado universitario de diferentes instituciones de educación superior. Aunque esto es un avance, la ausencia de estudios sobre educación musical no formal enfocada a la discapacidad visual en el país es insistente. Debido a esto, a continuación se realiza la revisión de referentes internacionales en torno al tema.

Uno de los intereses de los pedagogos es el de encontrar metodologías y guías didácticas para la enseñanza de la música a personas con discapacidad visual. A ello aportan Chaves Giesteira (2013), con el trabajo La enseñanza de la música para personas con discapacidad visual: elaboración y evaluación de un método de guitarra; y Sánchez (2017) con La didáctica del violín en alumnos con discapacidad visual: compendio de buenas prácticas, recursos y estrategias metodológicas para una educación inclusiva en las enseñanzas elementales y profesionales de música. Ambos estudios son tesis de posgrado realizadas en España, que abordan el aprendizaje de dichos instrumentos en relación con las herramientas didácticas y pedagógicas que pueden implementarse en las clases de estudiantes con discapacidad visual. También realizan una propuesta del aprendizaje de la musicografía, y la importancia del vínculo de ambos procesos (lectura musical e instrumento) en el proceso enseñanza-aprendizaje de la música en personas en esta condición.

En sintonía con las investigaciones en Colombia antes mencionadas, se encontraron referencias acerca de la enseñanza de la música en la escuela regular y en la educación superior. Uno de los estudios encontrados se trata de La enseñanza de la música a estudiantes deficientes visuales en el ámbito escolar convencional, de Clark y Murphy (1999), el cual pretende analizar la información que puede necesitar el profesor de música para responder a las necesidades de alumnos discapacitados visuales en el aula ordinaria. En el ámbito de la educación superior, se halla un estudio realizado por Chávez y Chávez (s.f.): La inclusión de personas con discapacidad visual en instituciones educativas musicales. Esta investigación tuvo la finalidad de analizar la inserción de estudiantes con discapacidad visual en instituciones musicales, su vinculación con las comunidades educativas y los recursos a los que tienen accesibilidad. En ella participaron personas con discapacidad visual de Argentina, Estados Unidos, Reino Unido, España, Estonia, Malasia, Panamá, República Dominicana y Suiza, y los resultados tienen resonancia con los de la investigación realizada en Colombia por Cano Hoyos (2016), más arriba explicada: aunque no se evidencian graves dificultades por parte de instituciones educativas musicales a la hora de admitir el ingreso de alumnos con discapacidad visual, la metodología de enseñanza no es adecuada para 
estas personas en todos los casos, no se cuenta con los software informáticos ni la accesibilidad tecnológica, ni cuentan con la bibliografía y el material musicográfico necesario para el estudio de la música.

Además, registra que en algunos de los reconocidos centros educativos del mundo (como el Trinity College de Londres en Reino Unido y en las universidades estadounidenses de New York, Brigham Young, y Las Vegas), se realiza un esfuerzo real por proporcionar al estudiante ciego las herramientas tanto tecnológicas como el material de estudio musicográfico, constituyendo de este modo una auténtica inserción del estudiante con discapacidad visual en el ámbito educativo musical. En contraparte, fuera de los centros urbanos no se cuenta con la misma accesibilidad, al punto de que varias personas en esta condición no pudieron participar de la investigación por no tener acceso a la tecnología (las encuestas se enviaban vía correo electrónico).

Finalmente, se trae a colación algunos estudios relacionados con el poder incluyente de la música para la persona con discapacidad visual, tales como los desarrollados por Arter y Hill (2000), Escuchar atentamente: ventajas derivadas de enseñar música a estudiantes discapacitados visuales; Rendón Nieblas (2015), La música como medio de aprendizaje: una experiencia sensorial incluyente; Macías y Antúnez (2015), Educación inclusiva: música para alumnos invidentes y débiles visuales; y García Carrascal (2016), Proceso de inclusión educativa y social en el ámbito musical de personas con discapacidad visual. En dichos estudios se esbozan las implicaciones que la enseñanza de la música tiene para el aprendizaje de otras áreas de conocimiento en los estudiantes con discapacidad visual, se presentan experiencias que demuestran su función terapéutica para la adaptación a la ceguera, como oportunidad de igualdad y reconocimiento, y se analiza el impacto de la tecnología en el aprendizaje musical de los estudiantes. También se concluye que existen carencias en la adaptación curricular y en la necesidad de la capacitación a docentes tanto al nivel básico como superior, tarea que, se propone, debe llevarse a cabo de manera interdisciplinar.

En síntesis, se puede concluir que los referentes investigativos en torno a la práctica de la educación musical de personas con discapacidad son pocos, y que se devela la ausencia de referentes investigativos que se acerquen al fomento de la autodeterminación de las personas con esta condición a partir de prácticas musicales no formales, tanto en el panorama local como en el regional e internacional. Por ello, los esfuerzos investigativos y académicos que se realicen para generar conocimiento sobre esta problemática específica siempre serán un aporte.

\section{Evolución del concepto de autodeterminación a partir del modelo social de la discapacidad}

A lo largo de la historia, la mirada hacia la discapacidad se ha observado con diferentes lentes que afectan directamente la percepción acerca de las posibilidades de desarrollo 
y participación de estos sujetos en la sociedad, así como la concepción sobre sí mismos. Según Palacios (2008), desde la antigüedad, la discapacidad había sido considerada con el modelo de prescindencia, el cual la justificaba desde el ámbito religioso: una maldición, un castigo divino, un cuerpo y mente defectuosos que no podían aportar nada a la sociedad; una carga para arrastrar. La vida de una persona con discapacidad no valía la pena ser vivida, postura que condujo a prácticas eugenésicas y marginación.

Solo hasta principios del siglo XX, la discapacidad es vista desde el modelo rehabilitador, el cual la consideraba una enfermedad que podía ser curada por medio del tratamiento médico y terapéutico. Vivirla era someterse a procesos de rehabilitación que pretendían normalizar a la persona hasta que pudiera nivelarse a las dinámicas sociales de la época. Esto era una tragedia personal y un problema social. Bajo esta mirada, la persona que vive en tal condición puede aportar a la sociedad en relación con el nivel de rehabilitación a la que se someta, por cuanto la educación especial surge como herramienta indispensable de este proceso. (Palacios, 2008). Si bien este modelo permitió un avance en la integración de esta población a la sociedad, se centró en las actividades que la persona no es capaz de realizar, generando una actitud paternalista, y el supuesto de discapacidad como sinónimo de inferioridad.

Más adelante, en los años 60, las personas con discapacidad en Estados Unidos e Inglaterra impulsaron acciones políticas por iniciativa propia, para "condenar su estatus como ciudadanos de segunda clase" (Palacios, 2008, p. 106), reorientando la atención gubernamental hacia las barreras sociales y ambientales que limitaban el desarrollo de su vida, la accesibilidad a servicios y recursos, y denunciaron las actitudes discriminatorias.

Posteriormente, en los años 70, hace su aparición el principio de la normalización, en el que se considera que las personas con discapacidad podían "conseguir una existencia tan normal como fuera posible" (Nirje 1972, citado en Torres y Peralta, 2006, p. 3). La aparición de este principio influyó poderosamente en el enfoque de servicios, la participación comunitaria y en los movimientos de autogestión de las personas con discapacidad.

Este escenario permitió que, entre los años 80 y 90, las personas con discapacidad defendieran su capacidad para hablar por sí mismos cuando se tomaban decisiones que afectaban sus vidas. Así comenzaron los grupos de autoayuda, que inician en Europa y se extienden luego hacia América, cuyos planteamientos proponían hablar por sí mismos, tener la posibilidad de adquirir conocimientos y habilidades para tomar decisiones acerca de su propia vida y participar visiblemente en la comunidad. Este movimiento, junto con los del movimiento de vida independiente y los grupos de autodefensa, fue determinante en la creación de una nueva conciencia social sobre las capacidades y los derechos de las personas con discapacidad (Peralta y González-Torres, 2006). 
Esta acción política generó un cambio de paradigma en cuanto a la visión que se tiene acerca de la discapacidad, denominado modelo social de la discapacidad, el cual "remarca que las causas que originan la discapacidad no son individuales -de la persona afectada-, sino sociales, por la manera en que se encuentra diseñada la sociedad" (Palacios, 2008, p. 103).

El modelo social de la discapacidad defiende dos fundamentos principales: en primer lugar, "las causas que originan la discapacidad no son religiosas ni científicas" (Palacios 2008, p. 25), como era la consideración de los modelos anteriores. Este afirma que la sociedad debe reconocer sus limitaciones para prestar servicios y asegurar adecuadamente que las necesidades de las personas con y sin discapacidad sean tenidas en cuenta dentro de la organización social (económica, cultural y ambiental). De esta manera, el problema no es individual ni exclusivo de la persona con discapacidad; es la sociedad la que ha fracasado al reconocer la diferencia y ajustarse a ella. El modelo promueve el principio de accesibilidad universal: que todos los entornos, productos, bienes y servicios cumplan los requisitos para poder ser utilizados por todas las personas (Pastor, 2012).

En segundo lugar, el sujeto con discapacidad es visto desde la capacidad: las personas con discapacidad tienen mucho que aportar a la sociedad y en la misma medida que aquellas sin discapacidad, puesto que su vida tiene igual sentido que la de cualquier otro sujeto. Así, el modelo se centra en lo que existe en el ser, en lugar de lo que hace falta. Esta nueva mirada genera cambios en las políticas públicas, legislaciones, y fomenta el activismo por medio de la creación de movimientos organizados y liderados por personas con discapacidad (Peralta y González-Torres, 2009).

Es en este contexto histórico y epistemológico que la autodeterminación se convierte en un concepto clave para la comprensión de los sujetos con discapacidad, puesto que "los individuos están intrínsecamente motivados a ser autodeterminados" (Peralta y González-Torres, 2009, p. 182). Su importancia universal defiende un constructo social que permite a todo individuo gozar de la posibilidad de elección y decisión como persona digna, incluso si tiene alguna discapacidad. Junto a los conceptos de autoestima, autoconfianza y calidad de vida, la autodeterminación se ha convertido en uno de los aspectos más importantes para la investigación y el estudio de la discapacidad (Peralta y González-Torres, 2009).

La autodeterminación es un concepto que posee dos acepciones, según Huges y Agran (1998, citado por Peralta y González-Torres, 2009), desde un sentido político, se refiere al derecho de un pueblo a ejercer el autogobierno; y desde el sentido psicológico, se define como "atributo personal o disposición a tener control sobre la propia vida y destino, es decir, a ser agente causal en la propia vida” (p.181). El concepto es utilizado por primera vez en la filosofía y las ciencias políticas del siglo XVII, visto como un principio de justicia que emerge de las naciones, constituidas desde su soberanía. A principios del siglo XX, la psicología de la personalidad lo acoge, enfocándose hacia el determinismo y la libertad de la conducta humana, y lo extiende hasta los estudios de 
motivación intrínseca, en los que se convierte en un concepto central en el abordaje de la discapacidad, desde que Nirje en 1972 se refiriera a "la autodeterminación como una faceta central del principio de normalización" (Torres y Peralta, 2006, p. 2). El postulado de Nirje estimuló la investigación, el diseño de instrumentos y currículos especializados alrededor del tema y su aplicación en los servicios y la educación para las personas con discapacidad. (Peralta y Arellano, 2014; Peralta y González-Torres, 2009; Verdugo, s.f.).

Como término, la autodeterminación surge a partir de los años 90, principalmente, como resultado de las investigaciones de Michael Wehmeyer, quien ha desarrollado el concepto de acuerdo con las revisiones de sus estudios y las de sus colaboradores. En la actualidad, el concepto de autodeterminación mayormente aceptado y difundido se refiere a "acciones volitivas que capacitan al individuo para actuar como el agente causal primario de su propia vida y para mantener o mejorar su calidad de vida, sin influencias o interferencias externas innecesarias" (Wehmeyer, 2006, citado en Tamarit, 2001, p. 24). Ser autodeterminado es una "característica disposicional de la persona" (Verdugo, s.f., p. 4 ).

\section{Autodeterminación como práctica}

De acuerdo con Verdugo (s.f.), Tamarit (2001), Abery (2003) y Wehmeyer \& Abery (2003), una acción se identifica como autodeterminada cuando contiene cuatro características esenciales: a) la autonomía, entendida como la capacidad de actuar según sus propias preferencias, intereses, y/o capacidades; b) la autorregulación (autodirección, control personal), vista como la habilidad para analizar un ambiente y un posible repertorio de respuestas para desenvolverse en ese ambiente, tomar decisiones sobre cómo actuar, cuánto control ejercer, cuándo y a quién cederlo y bajo qué circunstancias; c) el fortalecimiento-capacitación psicológica (empowerment), relacionado con las dimensiones del control percibido, entre las que se incluyen la eficacia personal y el descubrimiento de las propias capacidades; y finalmente, d) la autorrealización (autoconfianza), que se da cuando la persona actúa con la convicción de que es capaz de hacer lo necesario para conseguir los resultados esperados en un ambiente específico.

La modalidad de expresión de estas características varía con la edad, la oportunidad, la capacidad y el contexto (Wehmeyer et al., 2011). A medida que una persona avanza en el desarrollo de su ciclo vital puede adquirir los elementos necesarios para desarrollar dichas características por medio de acciones denominadas elementos o componentes de la autodeterminación, entre los cuales se incluyen el establecimiento de metas y objetivos, la adquisición de habilidades, la toma de decisiones y la resolución de problemas, las aptitudes de liderazgo y autogestión, el control interno que permita atribuciones positivas de eficacia, la comparación entre expectativas y resultados, la persistencia y el ajuste de los desafíos a la condición de discapacidad, entre otros. 
La autodeterminación es un derecho básico que se debe entender "como un derecho político" (Verdugo, s.f., p. 1), puesto que "es la garantía real para las personas, al margen de sus capacidades, de tener un proyecto vital, basado en su individualidad e identidad y ejercer control sobre el mismo" (Marcilla, 2012, p. 115). Sin embargo, cuando se refiere a la discapacidad, la garantía de dicho derecho tiende a ser vulnerado debido a preconcepciones sociales.En primer lugar,la discapacidad, del tipo quesea, sueleasociarse con la incapacidad para el ejercicio de la libertad. Esta dificultad puede acentuarse aún más en la realidad de las personas con discapacidad intelectual y psicosocial. En segundo lugar, algunas veces la sociedad no toma en serio las decisiones del sujeto con discapacidad porque supone una carencia de poder que limita su autocontrol y la calidad de sus decisiones sobre su propia vida. Sin embargo, la importancia universal de este derecho es su mejor argumento para defender la acción voluntaria de una persona. No confiar en la autodeterminación de un ser humano es negarlo (Verdugo, s.f.).

Wehmeyer y Metzler (1995, citados por Peralta y González-Torres, 2009) concluyeron que no es posible llevar a cabo conductas autodeterminadas si el contexto (familia, instituciones, comunidad) no ofrece oportunidades para su práctica. Es por esto precisamente que debe garantizarse el fomento de la autodeterminación en los sujetos, aún con mayor énfasis en las personas con discapacidad, por medio del acceso a apoyos efectivos que permitan el espacio, más amplio posible, de oportunidades de elección. La autodeterminación puede ser promovida, basándose en el principio esencial de "que toda persona, con independencia de su capacidad, puede mejorar su vida si se le ofrecen los apoyos adecuados" (Tamarit, 2001, p. 6), lo cual "requiere un cambio importante en la manera de concebir y planificar la provisión de apoyos escolares, sociales y familiares, prestando atención a los roles a desempeñar por los distintos agentes en cada uno de estos contextos" (Peralta y Arellano, 2014, p. 67). La autodeterminación, como acción fomentada y practicada, permitirá una adultez con mayores logros en cuanto a la autonomía, la vida independiente, la empleabilidad, el bienestar general, en resumen, una mejor calidad de vida de las personas con discapacidad, de acuerdo con Verdugo (s.f.), Peralta y Arellano (2014), Abery (2003) y Tamarit (2001).

\section{Educación musical e inclusión}

Teniendo en cuenta las ideas expuestas, se hace necesario resaltar la importancia que tiene la promoción de la autodeterminación en las personas con discapacidad a lo largo de su vida, y es a partir de ella que surge la respuesta a la pregunta orientadora del presente artículo, en el que se pretende analizar cuáles son los aportes que la educación musical puede ofrecer a las personas con discapacidad visual para promover conductas autodeterminadas. En el siguiente apartado se revisarán las transformaciones que la sociedad ha realizado en el paradigma de la discapacidad, las cuales han permitido que la educación musical incluya a las personas con discapacidad en procesos musicales no formales, como es el caso del programa Música para ver. 
Según Gainza (2003), la educación musical ha tenido una transformación a lo largo del siglo anterior. Los cambios sociales que modificaron la manera de enseñar impregnaron también a una educación musical estricta, de conservatorio, donde el fin era el sonido en sí mismo, mas no el sujeto que lo producía. Durante los años 80, la educación musical latinoamericana realizó un giro pedagógico importante. Luego de décadas de seguir estrictamente modelos musicales europeos, como los métodos Dalcroze, Orff, Willems, entre otros, en Argentina en los 80 se creó el movimiento "Pedagogías musicales abiertas", inspirado en los pensamientos del compositor y pedagogo Murray Schafer, y los principios pedagógicos de Freire.

La apuesta de esta postura pedagógica musical alternativa estaba basada en:

una especie de protesta mediante una acción personalizada, contestataria de la tradición, que revaloriza lo cotidiano, tradicionalmente segregado de la escuela y relegado a los espacios del tiempo libre y la educación no formal [con el objetivo de] liberar el pensamiento y la expresión (Gainza, 2003, p. 14).

La autora explica que el movimiento pedagógico pretendía concordar con la necesidad real de los niños y jóvenes de la época en cuanto a su desarrollo musical, promoviendo currículos flexibles y prácticas musicales creativas que hicieran uso de la experimentación y el descubrimiento personal y grupal, como las condiciones naturales para crecer musicalmente. Se interesó además por desarrollar en los futuros educadores musicales y en los estudiantes de música "la capacidad de libre reflexión, que les permitiera elegir y optar libremente" (Gainza, 2003, p. 20). El saber político que los sujetos consolidan por medio de la educación musical se prima sobre la técnica, la interpretación, el repertorio y el instrumento.

Así, la música y su enseñanza se convirtieron ahora en el medio y no en el fin. Se recuperaron las melodías de los ancestros y se dio valor a la música folclórica en las aulas, se permitió la creación musical a partir de los niños, y no solo de los libros. La propuesta se extendió por Latinoamérica, transformando durante las décadas siguientes la enseñanza musical, propiciando la investigación y creación de metodologías de educación musical interactivas, permitiendo el reconocimiento que en la actualidad se le otorga a la educación musical como estrategia de transformación social.

En este punto es preciso resaltar que la temporalidad de la recién explicada transformación que la educación musical experimentó concuerda con la época de transición del paradigma de rehabilitación al paradigma del modelo social de discapacidad, los movimientos sociales y políticos hacia el reconocimiento de la autodeterminación de las personas con discapacidad, y el avance en investigación y ampliación de servicios hacia la discapacidad bajo esta mirada, proceso detallado en la primera sección de este escrito. Todos estos cambios insinuaron nuevos desafíos a los gobiernos y sociedades acerca del abordaje de la discapacidad, y exigieron la atención de los líderes mundiales hacia el reconocimiento de la discapacidad como asunto de derechos humanos. Finalmente, la Convención de las Naciones Unidas sobre los derechos de las personas con discapacidad en el 2006 retó a los países miembros 
a adecuar, planear, implementar y ejercer control de sus políticas y prácticas hacia la discapacidad. Según Pichott y Vélez (2009), en Colombia los principios de dicha convención fueron recientemente aprobados a través de la Ley 1346 de 2009, que, amparada por el artículo 13 de la Constitución Política de 1991, se compromete a garantizar la inclusión de todas las personas

en los diferentes espacios de la sociedad, considerando [...] al ser humano como un ser con derechos y deberes, los cuales deben ser conocidos, asumidos y respetados, para generar un ambiente adaptado y estable para la persona con discapacidad y una relación dialéctica entre el sujeto y el entorno, que promueva un proceso dinámico de adaptación y competencia ocupacional y/o social (Céspedes 2005, citado en Castro et. al., 2013, p. 48).

De esta manera, la inclusión de las personas con discapacidad al aula escolar se convirtió en una ley, la cual garantiza el acceso de la población a la educación formal y no formal y la adaptación de currículos, recursos y metodologías de acuerdo con la particularidad:

Ante esta realidad, instituciones de base comunitaria, organizaciones no gubernamentales y gubernamentales han generado espacios que promueven la inclusión de estos grupos sociales, desde propuestas centradas en el reconocimiento de derechos fundamentales y la generación de espacios que facilitan la participación social en escenarios laborales, escolares, religiosos, artísticos y culturales (Castro et. al., 2013, p. 48).

Como resultado del cumplimiento de esta ley, y de las nuevas miradas que se construían al respecto de la educación musical en Latinoamérica, las personas con discapacidad empezaron a ganar terreno en la participación de formación musical en Colombia. Procesos como el de la Fundación Nacional Batuta involucraron a 200 niños, niñas y adolescentes con discapacidad a sus preorquestas a nivel nacional (Castro et. al., 2013); la Fundación Colsalud de la ciudad de Pereira, según se lee en su sitio web, desde el año 2005 creó el Grupo Talentos Colsalud, integrado por 110 niños, niñas, jóvenes y adultos con diferentes discapacidades (https://colsalud.org.co/talentoscolsalud/), entre otras iniciativas. Aunque aún muchas personas con discapacidad que no pueden acceder a una oferta educativa integral que garantice su derecho a la educación, los esfuerzos se amplían y lentamente se cierra la brecha y se transita hacia la verdadera inclusión (Meléndez, 2002). Es así como "hoy en día puede entenderse la música como un medio para la inclusión social de la población con discapacidad, ofreciendo un escenario que potencia capacidades de acuerdo con los intereses de la persona" (Castro et. al., 2013, p. 47).

\section{Música para ver}

Bajo estos nuevos paradigmas históricos acerca de la discapacidad y la educación musical surge la iniciativa Música para ver, programa de educación musical inicial ofrecido a niños, niñas, adolescentes, jóvenes y adultos con discapacidad visual (ceguera y baja visión), basado en el aprendizaje de las flautas dulces, el desarrollo 
vocal, el aprendizaje de musicografía braille y la práctica de un instrumento musical principal a elección del participante. El objetivo de Música para ver es ofrecer un espacio de educación musical de alta calidad para el desarrollo de sus capacidades musicales y ampliar su proyección de vida.

El programa es liderado por la Fundación Uno más Uno, en cabeza de su director ejecutivo, Luis Fernando Gómez, quien realizó un tránsito profesional de empresario del sector de la moda a empresario social, luego de perder la visión a causa de una enfermedad visual degenerativa denominada retinitis pigmentosa. A partir de ese momento, su misión de vida fue aportar a la detección temprana de problemas visuales en los niños y niñas de Medellín y el Oriente Antioqueño. Más adelante, encontró la forma de unir su misión con su pasión, la música, realizando conciertos de recolección de fondos en asocio con la Orquesta Filarmónica de Medellín. En 2014, en alianza con la Fundación Incolmotos Yamaha, Música para ver se concibió como programa educativo musical no formal. En la actualidad se benefician más de 500 niños, niñas, adolescentes, jóvenes y adultos en el país.

El camino recorrido, aunque corto, ha sido suficiente para develar las capacidades de los participantes del programa, en todo su colorido abanico de posibilidades. En las siguientes líneas se analiza cómo esta práctica musical fomenta en ellos acciones autodeterminadas.

\section{Música para ver, como práctica para el fomento de la autodeterminación}

Aunque la autodeterminación de las personas con discapacidad es uno de los temas acerca de los que se investiga en la actualidad (Peralta y Arellano, 2014), en el rastreo de bibliografía para la construcción del presente artículo se hizo evidente la ausencia de estudios acerca de los aportes de la educación musical en la promoción de conductas autodeterminadas en las personas con discapacidad. Dada la experiencia educativa de Música para ver, y de acuerdo con los resultados develados en la sistematización de experiencias de la cual hace parte este artículo, se considera pertinente analizar cómo la práctica musical colectiva favorece el desarrollo de la autodeterminación en niños, niñas y adolescentes con discapacidad visual. El siguiente relato sirve a tal propósito.

El programa tuvo una experiencia singular con un grupo de 20 niños y niñas con discapacidad visual entre los 9 y 13 años de edad, estudiantes de la Institución Educativa Francisco Luis Hernández Betancur, antiguo Colegio de Ciegos y Sordos de Medellín (CIESOR). El grupo había tenido un proceso de dos años de interpretación de flautas dulces, bajo la responsabilidad pedagógica de la autora de este texto, quien era en ese momento su docente. Metodológicamente, el grupo había recorrido un camino musical desde un repertorio muy sencillo al unísono hasta arreglos a tres líneas melódicas y canto con acompañamiento armónico y percusivo. También había realizado varias 
presentaciones musicales dentro de su institución, los cuales se veían como espacios para el reconocimiento de sus habilidades. Sin embargo, tanto el acompañamiento instrumental como la selección y adaptación de repertorios y las dinámicas de clase habían sido propuestos y liderados por la docente.

En determinado momento el grupo se enfrentó a un reto novedoso: recibió la invitación para realizar una presentación musical fuera de la institución, en un horario en el que su docente no podía acompañarles. Discutieron varias semanas, intentando determinar la respuesta a la invitación. ¿La rechazaban? Era una gran oportunidad, pero si la tomaban, ¿cómo realizarían la presentación sin la docente? ¿Sería necesario buscar ayuda, alguien que la reemplazara? ¿Proponían otra fecha? ¿Quién se encargaría de la logística, y de la movilidad de los niños y niñas en un espacio que desconocían?

Finalmente, el interés personal y colectivo por la música les permitió tomar la decisión: "vamos a tocar". Eligieron un repertorio conocido y propusieron como posibles directores a algunos niños pertenecientes al grupo, con el objetivo de que estuviesen en frente de cada canción, dirigiendo desde el piano o la percusión. Buscaron a dos adultos que los acompañaran para colaborar con su movilidad. Planearon la ubicación en el escenario y practicaron varias veces el programa musical, poniendo atención a cada detalle. Al llegar el día, realizaron la muestra musical tal como lo planearon. A la siguiente clase, la euforia era total. Su felicidad radicaba en que le demostraron a su docente de música que ya no necesitaban de ella para realizar una presentación musical. A partir de ese momento, cada vez que hay una oportunidad se les motiva para que ellos gestionen, lideren y propongan nuevas presentaciones, elijan el repertorio, y modifiquen las interpretaciones musicales con libertad. Ahora la docente es su colaboradora, su acompañante, su ayuda. No dependen de ella.

Este ejemplo describe solo un momento, de muchos otros, en los que cotidianamente se genera la oportunidad para el desarrollo de la autodeterminación dentro de las clases de música del programa, en el que se hace notorio el ejercicio de la autonomía, la autorregulación, el empoderamiento y la autoconfianza. El proceso educativo musical fomenta estas características de la autodeterminación en los estudiantes porque se basa en cinco principios, que se explican a continuación.

En primer lugar, una de las principales actividades que permiten el desarrollo de la autodeterminación en los niños, niñas y adolescentes de Música para ver es la participación en la planificación del proceso educativo en un entorno que valora la colectividad, lo que según Abery (2003) y Wehmeyer \& Abery (2013) mejora la oportunidad de elección, en comparación de los procesos educativos individuales y poco participativos. Cuando un sujeto se enfrenta al aprendizaje de un instrumento musical, la acción misma de interpretarlo modifica sustancialmente sus propias concepciones sobre sí, sobre los otros, y sobre el nosotros, puesto que uno de los principios de Música para ver es el aprendizaje en grupo. Se observa en las clases que poco a poco, mediante la interacción con el instrumento y con los pares, se tejen significados que hacen aflorar la participación personal y colectiva. 
En segundo lugar, la oportunidad de aprender una nueva habilidad es uno de los indicadores que está directamente relacionada con el desarrollo de la autodeterminación. Según las investigaciones de Wehmeyer, Abery, Mirhaug \& Stancliffe (2003), las personas con discapacidad "tienen significativamente menos oportunidades de aprender, practicar y refinar las capacidades personales relacionadas con la autodeterminación, por lo que las medidas de la capacidad pueden estar enmascarando cuestiones relacionadas con la oportunidad" (p. 9). Música para ver se ha constituido en uno de los apoyos que permite la exploración y el desarrollo de las habilidades musicales y artísticas de los participantes, con metodologías adaptadas a sus necesidades, sin restricción de edad ni de discapacidades asociadas.

En tercer lugar, la autodeterminación es fomentada cuando se permite que el sujeto sea consciente de su poder de ser autónomo. Ese descubrimiento de sí mismo es subjetivo, cada ser encuentra su autonomía de diferentes formas. En Música para ver, la música ha sido la herramienta, la estrategia, que ha permitido a los participantes reconocer el sonido como una nueva forma de verse a sí mismos y a su entorno, una ventana para leer su realidad, para ser, hacer y estar en el mundo, y de visualizar su futuro. Además, el placer de la interpretación instrumental y vocal ha sido un detonante en la búsqueda de la autonomía que los motiva a superar los obstáculos que el entorno ha impuesto sobre su condición, tanto en la movilidad y el acercamiento a espacios desconocidos como en la toma de decisiones basadas en sus intereses. De acuerdo con Wehmeyer \& Abery (2013) "hay evidencia suficiente para apoyar las intervenciones para enseñar o promover la toma de decisiones, la resolución de problemas, el establecimiento de metas y logros, y las habilidades de autodefensa como prácticas efectivas para promover la autodeterminación" (p. 9).

En cuarto lugar, recibir un instrumento musical conlleva a la práctica del uso del derecho y del deber: como sujeto, el participante es consciente del privilegio que tiene al poseerlo, explorarlo y aprovecharlo, a la vez que reconoce la exigencia del cuidado, la protección y la responsabilidad de darle el uso correcto al tocar (Gómez, 2009). Luego de la práctica cotidiana, la significación de esa posesión se demuestra en el sentimiento subjetivo hacia él, convirtiéndolo en parte de su nueva dinámica personal y generando una opción en el agenciamiento de su tiempo libre (Tamarit, 2001). Aceptar este proceso, asumirlo encuentro tras encuentro, día tras día, fortalece su autorregulación, a medida que la práctica musical se hace continua.

En determinado momento, al tocar frente al público, la música y el instrumento se convierten en un nuevo canal de comunicación y placer (Gómez, 2009). Ese corto espacio de reconocimiento estimula y refuerza en el sujeto el ejercicio de autodeterminación, la cual ejerció cada día, eligiendo en la cotidianidad uno u otro camino, para persistir en la ejecución del instrumento, y le hace proponerse nuevos 
retos musicales, que a su vez generan otros niveles de exigencia y nuevas opciones para decidir. Esto halla resonancia con lo que Tamarit (2001) denomina "Plan personal de progreso", el cual permite el desarrollo de la autodirección como habilidad central en el desarrollo personal y la autodeterminación en un sujeto, posibilitando el ajuste de las expectativas personales y las disposiciones contextuales en la consecución de sus metas. La continuidad de este ciclo convierte sus capacidades en potencialidades, transformando su mirada hacia las diferencias orgánicas, psicológicas, emocionales y sociales que están implícitas en su condición de discapacidad.

El proceso de equilibrar los intereses personales y lograr la armonía del colectivo en esa cotidianidad le permite reconocerse igual a todos, con capacidad de crear, sentir e interpretar, y a la vez único, importante, partícipe (Gómez, 2009). Para un grupo humano cuya voz está siendo apenas reconocida en la sociedad colombiana, como es el de las personas con discapacidad, este encuentro se torna aún más valioso y permite un tránsito de la dependencia hacia la interdependencia: "Cada músico [...] es él con su instrumento, pero todos dependen de él, y él a su vez depende de todos los otros" ( p.662 ). En Música para ver, los participantes realizan actos espontáneos que aportan a la consecución de una meta individual y colectiva, y se estimula la búsqueda y ofrecimiento de apoyo ante una acción, lo que es un indicador de acciones autodeterminadas, según Tamarit (2001).

En conclusión, la pertenencia a un proceso musical juega un papel importante en las personas con discapacidad visual porque no solo las empodera, sino que también las sitúa en una perspectiva proyectiva, modificando su visión de futuro y promoviendo la persistencia en el cumplimiento de sus metas y planes deseables.

\section{Un ambiente educativo y musical que estimula la autodeterminación}

La práctica musical colectiva, cuando es propiciada en un ambiente creativo y sensible, cercano a los principios pedagógicos de las "pedagogías musicales abiertas", como es el caso de Música para ver, puede fomentar momentos pedagógicos y artísticos para enfrentar el propio temor y el impuesto por el entorno, especialmente el que ha prevalecido en la condición de discapacidad. Hacer música está directamente ligado a la independencia, a la búsqueda de su propia interpretación. El autoreconocimiento de los logros musicales crea conciencia de la necesidad de ayuda, mas no de una dependencia absoluta.

Un ambiente educativo musical que fomenta la autodeterminación es aquel que permite relaciones horizontales, significativas y recíprocas, que valora las subjetividades con su autenticidad, que promueve la solidaridad, que es propositivo, equitativo y comunicativo, que busca el respeto, el afecto y el vínculo, que permite el liderazgo de 
todos los actores, que ofrece la información por adelantado, que genera oportunidades y respeto por la diversidad, que estimula la participación en un contexto libre de amenaza y que mantiene la autoestima de los involucrados (Gadberry \& Harrison, 2016; Tamarit, 2001).

El docente de música que desee propiciar la autodeterminación en su ejercicio musical y educativo debe ser partidario de ella, dar la oportunidad a los músicos en formación de ejercer control, asegurarse de que asuman las responsabilidades y experimenten las consecuencias positivas y negativas de sus decisiones, promover la comunicación de lo que están pensando al tomar una decisión y evaluarla, entre otras actitudes (Abery, 2003):

Los profesores son sin duda los principales agentes educativos para promover la conducta autodeterminada. Sus creencias y actitudes se reflejan en el modo de enfocar su práctica docente y su motivación para formarse y poner en marcha las estrategias didácticas que son necesarias para facilitar esta conducta (Peralta y González-Torres, 2009 p. 187).

\section{Conclusiones}

Retomando el objetivo inicial de la sistematización de experiencias en la que se basa el presente artículo de reflexión (Pérez, Ruiz y Ossa, 2019), y en relación con los objetivos de esta investigación, se presentan las siguientes conclusiones.

En primer lugar, el estudio de la música

permite a las personas con discapacidad visual adquirir disciplina y autoconfianza, elementos claves para la construcción de subjetividad, en la medida en que se reconocen como sujetos con discapacidad visual, que sobrepasan su condición de ciegos para potenciar el horizonte de su propia existencia en la búsqueda de autonomía (Pérez, Ruiz y Ossa, 2019, p. 64).

El concepto de autodeterminación referido en este artículo complementa esta afirmación, dado que, en el caso particular de Música para ver, el estudiante tiene la posibilidad de ser consciente de su poder de ser autónomo, del uso del derecho y del deber, y de ejercer control sobre su propio proceso de aprendizaje, tomando decisiones de acuerdo con sus propias preferencias, intereses y capacidades. Esta posibilidad aporta al desarrollo de su autonomía.

En segundo lugar, la sistematización concluye que "una práctica pedagógica como Música para ver (...) favorece la exploración de los intereses particulares de sus participantes en un ambiente de colectividad" (Pérez, Ruiz y Ossa, 2019, p. 65), permitiendo el ejercicio de equilibrar los intereses personales con los intereses del colectivo y propiciando espacios de reconocimiento y participación, pilares esenciales del fomento de la autodeterminación. 
Finalmente, "Música para Ver permite a los participantes del programa desarrollar habilidades encaminadas a la realización de sus proyectos de vida alrededor de la música, ya que se visualizan a futuro como artistas profesionales" (Pérez, Ruiz y Ossa, 2019, p. 65), lo que en relación con el concepto de la autodeterminación estimula el ejercicio de la autodirección, autorregulación y autoconocimiento.

\section{Reflexiones finales}

A pesar de ser uno de los temas que genera interés investigativo en la actualidad, según Wehmeyer \& Abery (2013), la autodeterminación de las personas con discapacidad requiere aún de la exploración investigativa en algunos aspectos, tales como el impacto de las intervenciones sociales y educativas en diferentes culturas de acuerdo con su etnia y lengua, los impactos del ambiente familiar en las acciones autodeterminadas y la autodeterminación en niños en edad escolar y en personas adultas. En este aspecto, se considera importante realizar estudios empíricos acerca del impacto de la educación musical y/o artística en la autodeterminación de las personas con discapacidad en sus diferentes momentos vitales.

Finalmente, es significativo resaltar la necesidad de realizar futuros procesos investigativos y reflexivos hacia la enseñanza de la musicografía braille y su impacto en la autodeterminación de las personas con discapacidad visual que deciden su proyecto de vida hacia la música, lo cual toma mayor relevancia al considerar el poco material musicográfico existente, las escasas propuestas pedagógicas para este tipo de escritura musical y el desconocimiento de los docentes de música al respecto.

La aspiración de este escrito ha sido aportar desde la academia, la investigación y la experiencia pedagógica de Música para Ver, una reflexión a los educadores musicales que inician la travesía de diseñar y adaptar programas musicales hacia la discapacidad visual en Colombia, que motive el diálogo de saberes y que provoque nuevas preguntas acerca de la educación musical en relación con el fomento de la autodeterminación.

\section{Referencias}

Abery, B. (2003). Autodeterminación y sordoceguera (Traducido por Karen María Berríos Mathie). San José, Costa Rica: . Escuela para Ciegos, Massachusets.

Andrade, A. (2013). Diseño de un software para el reconocimiento de símbolos matemáticos en latex mediante síntesis de voz para personas con discapacidad visual (Tesis de pregrado). Universidad Tecnológica de Pereira, Pereira, Colombia. Recuperado de http://recursosbiblioteca.utp.edu.co/dspace/handle/11059/4089 
Arias Cancino, H. D. (2015). Estrategias pedagógicas desde la inclusión educativa para el fortalecimiento de la expresión y la conciencia corporal para estudiantes de música con discapacidad visual. Recuperado de http://repository.pedagogica.edu.co/ handle/20.500.12209/1547

Arter, C. y Hill, D. L. (2000). Escuchar atentamente: ventajas derivadas de enseñar música a estudiantes discapacitados visuales. Entre dos Mundos: Revista de Traducción sobre Discapacidad Visual, (14), 5-12.

Castro, E., Bocanegra, K., Garzón, G., González, A., Hernández, H., Maldonado, H.,... Triana, D. (2013). Análisis bibliométrico sobre la inclusión de niños, niñas y adolescentes con discapacidad haciendo uso de la música como herramienta central. Ciencia y Salud, 11(1), 45-58.

Burgos, S. (2008). Educación física y discapacidad visual: Una resignificación de la vida. Revista Corporeizando, 11(1), 68-86. Recuperado de http://revistas.pedagogica. edu.co/index.php/corporeizando/article/view/2008

Cano Hoyos, V. (2016). Inclusión a personas con discapacidad visual en los pregradosy licenciaturas (Tesis demaestría).Universidad Pontificia Bolivariana,Medellín,Colombia. Recuperado de https://repository.upb.edu.co/bitstream/handle/20.500.11912/2940/ Tesis $\% 20$ maestri $\%$ C C $\% 81$ a $\% 20$ en $\% 20$ ed uca ci o \% C C $\% 81$ n $\% 20 \% 20$ Valenti\%CC\%81n\%20Cano\%20Hoyos.pdf?sequence=1\&isAllowed=y

Castillo Castellanos, J. y Delgado Villamizar, J. J. (2012). Transcripción al sistema braile de material básico para el estudio de la música (Tesis doctoral). Universidad Industrial de Santander, Santander, Colombia.

Chacón, M., Camargo, M. y Cotachira, A. (2014). Estrategia didáctica para la inclusión de jóvenes en situación de discapacidad visual a la clase de Educación Física del grado $1103^{\circ}$ en el Colegio José Félix Restrepo (Tesis de pregrado). Universidad Libre, Bogotá, Colombia. Recuperado de http://hdl.handle.net/10901/7626

Chaves Giesteira, A. (2013). La enseñanza de la música para personas con discapacidad visual: elaboración y evaluación de un método de guitarra (Tesis doctoral). Universitat Autònoma de Barcelona, Barcelona, España.

Chávez, P. G. y Chávez, F. (s.f.) La inclusión de personas con discapacidad visual en instituciones educativas musicales. Recuperado de https://www.chaveztwins.com/ LA\%20INCLUSI\%C3\%93N\%20DE\%20PERSONAS\%20CON\%20DISCAPACIDAD\%20 VISUAL\%20EN\%20INSTITUCIONES\%20EDUCATIVAS\%20MUSICALES[87].pdf

Clark, A. y Murphy, F. (1999). La enseñanza de la música a estudiantes deficientes visuales en el ámbito escolar convencional. Entre dos Mundos: Revista de Traducción sobre Discapacidad Visual, (10), 5-15. 
Correa, Y. y Pulido, E. (2013). Adaptación e implementación de recursos didácticos para la enseñanza de ecuaciones de primer y segundo grado a niños con discapacidad visual en un aula inclusiva. Educación Científica y Tecnológica, 510-514. Recuperado de http://revistas.udistrital.edu.co/ojs/index.php/revcie/article/viewFile/7714/9524

Fundación Social Colombiana de Salud COLSALUD https://colsalud.org.co/ talentos-colsalud

Gadberry, A. \& Harrison, A. (2016). Music therapy promotes self-determination in young people with autism spectrum disorder. International Journal of School \& Educational Psychology, 4(2), 95-98. 10.1080 / 21683603.2016.1130580

Gainza, V. (2003) La educación musical entre dos siglos: del modelo metodológico a los nuevos paradigmas. Serie "Documentos de Trabajo". Buenos Aires: Escuela de Educación Universidad de San Andrés.

Gainza, V. (2002). Pedagogía musical - Dos décadas de pensamiento y acción educativa. Buenos Aires: Editorial Lumen.

García Carrascal, S. V. (2016). Proceso de inclusión educativa y social en el ámbito musical de personas con discapacidad visual (Tesis de grado). Pontificia Universidad Católica del Ecuador, Pichincha, Ecuador.

Gómez, M. R. (2009). Batuta Caldas-Colombia: un programa de formación musical que deviene en formación ciudadana. Revista LGómezatinoamericana de Ciencias Sociales, Niñez y Juventud, 2(9), 649-668.

Hernández, C., Jiménez L., Juez G. y Galvis, J. (2008). El SEMLEB: Una herramienta para la enseñanza de la lecto-escritura en niños con discapacidad visual. Recuperado de https://www.redalyc.org/pdf/304/30400713.pdf Loaiza, C. y Torres, S. (2013). Horus: Prototipo de herramienta tecnológica de apoyo semilibre, para personas con discapacidad visual. Ventana Informática, 29, 13-28. Recuperado de http://revistasum. umanizales.edu.co/ojs/index.php/ventanainformatica/article/view/241/325

Macías, R. A. A. C. y Antúnez, S. Á. S.2015. Educación inclusiva: música para alumnos invidentes y débiles visuales. Una Visión Interdisciplinaria del Arte, 24-43.Meléndez, L. (2002). La inclusión escolar del alumno con discapacidad intelectual. Bogotá: GLARP-IIPD.

Palacios, A. (2008). El modelo social de la discapacidad: orígenes, caracterización y plasmación en la convención internacional sobre los derechos de las personas con discapacidad. Madrid: CERMI.

Pastor, C. (2012). Aportaciones del diseño universal para el aprendizaje y de los materiales digitales en el logro de una enseñanza accesible. Recuperado de http:// diversidad.murciaeduca.es/publicaciones/dea2012/docs/calba.pdf 
Peralta, F. y González-Torres, C. (2009). El movimiento hacia la autodeterminación personal: antecedentes y estado actual. Trabajo presentado en el XV Coloquio de Historia de la Educación: El largo camino hacia la una educación inclusiva: la educación especial y social del siglo XIX hasta nuestros días (pp. 181-192). Pamplona: Universidad Pública de Navarra.

Peralta, F. \& Arellano, A. (2014). La autodeterminación de las personas con discapacidad intelectual: situación actual en España. Revista CES Psicología, 7(2), 59-77.

Pérez Valderrama, M., Ruiz, N. y Ossa Fernández, C. (2019). Música para ver: Sistematización de la experiencia (Tesis de maestría). Universidad de Manizales, Manizales, Colombia.

Pichott, A. Velez, J. (2009) ¿Se garantiza el Derecho Fundamental a la educación a los niños en situación de discapacidad sensorial visual de la ciudad de Medellín en lo referido a los procesos de integración acceso y permanencia en la educación formal? (Proyecto de grado monografía). Universidad de Antioquia, Medellín, Colombia.

Rendón Nieblas, E. G. (2015). La música como medio de aprendizaje: una experiencia sensorial incluyente. Ponencia presentada en el Segundo Encuentro Nacional de Gestión Cultural (pp. 1-13).

Sánchez, L. S. (2017). La didáctica del violín en alumnos con discapacidad visual: compendio de buenas prácticas, recursos y estrategias metodológicas para una educación inclusiva en las enseñanzas elementales y profesionales de música (Tesis doctoral). Universidad Católica San Antonio de Murcia, Murcia, España.

Suárez Román, J. C. (2016). Percepción de la música en la discapacidad visual (Tesis de maestría). Universidad EAFIT, Medellín, Colombia.

Tamarit, J. (2001). Propuestas para el fomento de la autodeterminación en personas con autismo y retraso mental. En Verdugo, M. A. y Jordán de Urríes, F. B., (Eds.) Apoyos, autodeterminación y calidad de vida. Salamanca: Amarú Ediciones.

Torres, M. C. G. y Peralta, F. (2006). Autodeterminación y discapacidad: Antecedentes y estado actual de un nuevo paradigma en la educación especial. Revista Española de Pedagogía, 231, 275-288.

Torres, J. y Gaviria, Y. (2013), Una propuesta inclusiva para la representación geométrica de poliedros con población en condición de discapacidad visual. Educación Científica y Tecnológica, 32-36. Recuperado de http://revistas.udistrital.edu.co/ojs/ index.php/revcie/article/view/5479

Velasco, I. y Montes, E. (2013). Propuesta para la enseñanza del álgebra geométrica a estudiantes con discapacidad visual, a través de la adaptación de material inclusivo. En P. Perry (Ed.), Memorias del 21ํㅡㄹ Encuentro de Geometría y sus Aplicaciones (pp. 291298). Bogotá, Colombia: Universidad Pedagógica Nacional. 
Verdugo, M. (s.f.). Autodeterminación y calidad de vida en las personas con discapacidad. Propuestas de acción. Recuperado de http://ardilladigital.com/ DOCUMENTOS/CALIDAD\%20DE\%20VIDA/CALIDAD\%20DE\%20VIDA\%20Y\%20 BUENA\%20PRACTICA/Autodeterminacion $\% 20 \mathrm{y} \% 20$ calidad\%20de\%20vida\%20en $\% 20$ las\%20personas\%20con\%20discapacidad\%20-\%20Verdugo\%20-\%20articulo.pdf

Wehmeyer, M. (2011), "Assessment and intervention in self-determination", Scruggs, T. and Mastropieri, M. (Ed.) Assessment and Intervention (Advances in Learning and Behavioral Disabilities, Vol. 24), Emerald Group Publishing Limited, Bingley, pp. 213-249. https://doi.org/10.1108/S0735-004X(2011)0000024011

Wehmeyer, M. \& Abery, B. (2013). Self-Determination and Choice. Intellectual and developmental disabilities. 51(5), 399-411. Recuperado de https://www.researchgate. net/publication/259199778_Self-Determination_and_Choice

Wehmeyer, M., Abery, B., Mithaug, D., \& Stancliffe, R. (2003). Theory in selfdetermination: Foundations for Educational Practice. Illinois: Charles C Thomas Publisher. 